\title{
\begin{tabular}{l|l} 
Mitraries & DSpace@MIT
\end{tabular}
}

\author{
MIT Open Access Articles
}

\section{High Throughput Synthesis of Uniform Biocompatible Polymer Beads with High Quantum Dot Loading Using Microfluidic Jet-Mode Breakup}

The MIT Faculty has made this article openly available. Please share how this access benefits you. Your story matters.

Citation: Lee, Seung-Kon, Jinyoung Baek, and Klavs F. Jensen. “High Throughput Synthesis of Uniform Biocompatible Polymer Beads with High Quantum Dot Loading Using Microfluidic JetMode Breakup." Langmuir 30, no. 8 (March 4, 2014): 2216-2222.

As Published: http://dx.doi.org/10.1021/la4041198

Publisher: American Chemical Society (ACS)

Persistent URL: http://hdl.handle.net/1721.1/92772

Version: Author's final manuscript: final author's manuscript post peer review, without publisher's formatting or copy editing

Terms of Use: Article is made available in accordance with the publisher's policy and may be subject to US copyright law. Please refer to the publisher's site for terms of use. 


\title{
High Throughput Synthesis of Uniform
}

\section{Biocompatible Polymer Beads with High Quantum}

\section{Dot Loading Using Microfluidic Jet-Mode Breakup}

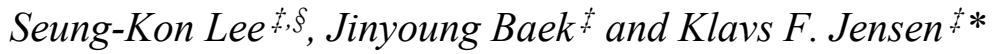 \\ + Department of Chemical Engineering. Massachusetts Institute of Technology, 77 \\ Massachusetts Avenue, 66-342, Cambridge, MA 02139 (USA)
}

KEYWORDS: microfluidics, jet breakup, drop breakup, hydrogels, quantum dots

§ Radioisotope Research Division. Korea Atomic Energy Research Institute, 989-111 Daedeokdaero, Yuseong-gu, Daejeon 305-353 (Republic of Korea) 
Abstract

Uniform polymer microbeads with highly loaded quantum dots (QDs) are produced using high-throughput coherent jet-breakup of a biocompatible polyethyleneglycol diacrylate (PEGDA) prepolymer resin, followed by in-line photo-polymerization. A spiraling and gradually widening channel enables maximum absorption of radiated UV light for the in-line photopolymerization without coalescence and clogging issues. Although the dripping-mode in general provides superior uniformity to the jet-mode, our nozzle design with tapered geometry brings controlled jet-breakup leading to $3 \%$ of uniform particle size distribution, comparable to dripping-mode performance. We achieve a maximum production rate of $2.32 \mathrm{kHz}, 38$ times faster than the dripping mode, at a same polymer flow rate. In addition, the jet-mode scheme provides better versatility with 3 times wider range of size control, as well as the compatibility with viscous fluids that could cause pressure build-up in the microsystem. As a demonstration, a QD-doped prepolymer resin is introduced to create uniform biocompatible polymer beads with $10 \mathrm{wt} \% \mathrm{CdSe} / \mathrm{ZnSe} \mathrm{QD}$ loading. In spite of this high loading, the resulting polymer beads exhibits narrow band width of $28 \mathrm{~nm}$ to be used for the ultrasensitive bio-imaging, optical coding and sensing sufficiently with single bead. 


\section{Introduction}

There has been intense and longstanding interest in the use of biocompatible fluorescent microparticles for a variety of applications such as bio-imaging ${ }^{1}$, drug delivery ${ }^{2,3}$, biological assays ${ }^{4,5}$, clinical diagnostics $^{6}$, optical encoding ${ }^{7}$, lasers $^{8}$ and display applications. ${ }^{9}$ Especially, particles with embedded quantum dots (QDs) have been received attention due to their useful optical properties. ${ }^{7,10}$ Batch synthesis has been the method of choice for preparing QD-doped microparticles due to practical advantages in productivity and accessibility, despite limitations such as poor loading, low throughput and material loss. In the batch loading processes, a significant portion of loading materials is wasted during the multistep process of synthesis, purification, loading, and washing. ${ }^{7,10}$ This is especially problematic when expensive QDs or biomaterials are used.

On the other hand, recent microfluidic droplet formation schemes (typically operating in the so-called dripping mode) allow production of uniform particles without the aforementioned issues. ${ }^{11-23}$ However in dripping-mode, the controllable size range is limited because the emulsion sizes are more dependent on the nozzle diameter and interfacial properties rather than the flow conditions. ${ }^{24}$ To get smaller emulsion sizes, dimensions of the nozzles at the breakup junction must be decreased, causing fabrication difficulties and significant pressure drop in the system. Use of viscous polymers or colloidal suspensions further increases pressure drops, which can cause device failures. A number of studies have presented ultrafast production of uniform or non-uniform water droplets in $1-10 \mathrm{kHz}$ range (See Table S1), ${ }^{17-21}$ but in-line synthesis of uniform polymer beads through breakup of viscous polymers has not been reported in such a high rate. Therefore, challenges still remain for improving the productivity, widening the controlled size range and achieving smaller size without severe pressure drop., ${ }^{3,42-24}$ At higher 
Weber number $(\mathrm{We})$ than corresponding to the dripping regime, the ejected liquid forms a cylindrical jet that breaks into tiny droplets by capillary instability. ${ }^{25-29}$ Formation of emulsions and particles using jet-breakup has been studied over several decades due to its significant practical advantage in mass production over the dripping scheme. ${ }^{25-29}$ However, difficulties in control of jet-mode uniformity have limited wide application to particle synthesis. ${ }^{29,30}$

In this study, we demonstrate production of uniform emulsions using high throughput harmonic breakup of a jet in a high shear co-flowing system with a well-designed nozzle. Since the jet diameter is relatively small and controllable for a given nozzle diameter, the jet-mode simultaneously provides superior control over a range of diameters, moderate pressure drops, and higher production rates, compared with the dripping-mode breakup. With this scheme, we achieve uniform fluorescent microbeads through in-line photopolymerization of biocompatible prepolymer resin containing about $10 \mathrm{wt} \% \mathrm{CdSe} / \mathrm{ZnSe} \mathrm{QD}$ nanocrystals. In spite of this high loading, photoluminescent spectra from the microbeads present narrow emission with band width of $28 \mathrm{~nm}$ without broadening or distortion of the peak shape. Unlike the batch synthesis, there are no additional washing steps required for the removal of unreacted residues causing dissipation of materials. ${ }^{4,9,31}$

\section{Experimental}

Preparation of microfluidic devices: The microfluidic chips were fabricated creating $100 \mu \mathrm{m}$ thick and $160 \mu \mathrm{m}$ thick SU-8 patterns by negative photolithography followed by polydimethyl siloxane (PDMS) embossing (PDMS 184-A and B, Dow Corning). The resulting PDMS replicas were bonded to $25 \mathrm{~mm}$ by $75 \mathrm{~mm}$ slide glass (Corning) covered with a thin PDMS film following oxygen plasma treatment of both surfaces. 
Flow rate control: Inner and outer fluids were introduced into the microfluidic device through Polytetrafluoroethylene (PTFE) tubing and the flow rates were controlled using syringe pumps (PHD Ultra, Harvard Apparatus) and gas tight syringes (SGE). Pressure drop of the core fluid was measured from the pressurized feeding system. The pressure range was from $0.5-3 \mathrm{~atm}$ when the overall flow rates vary from $3-100 \mu \mathrm{L} / \mathrm{min}$. Hexadecane (99\%, Sigma-Aldrich) with 4 wt $\%$ of surfactants (EM 90, ABIL) was used for the outer continuous phase.

System for UV exposure: A UV lamp (FluoroArc, 100W, Carl Zeiss) mounted on an inverted microscope (Axiovert 200, Carl Zeiss) was used for the in-line photo-polymerization of the prepolymer emulsions. The power of the UV light source was adjusted to $50 \mathrm{~W}$ for optimized production of the microparticles.

Laser and high-speed photography: A double frame high speed camera (Imager Intense, La Vision) and two ND:YAG laser (532 nm, $30 \mathrm{~mW}, 10 \mathrm{~Hz}, 7 \mathrm{~ns}$, Big Sky Laser) were used for laser photography analysis of jet-mode breakup. ${ }^{32}$ A fluorescent dye (Rhodamine 6G, SigmaAldrich) was used as a contrast agent for polyethyleneglycol diacrylate phase (PEGDA, MW 258, Sigma-Aldrich).

Interfacial tension measurement: Interfacial tension of PEGDA and hexadecane was measured using ring tensiometry (K100, Kruss) with 0.5 inch platinum ring.

\section{Result and discussion}

\subsection{Experimental scheme}

The high-throughput microfluidic particle preparation system (Figure 1) is composed of two functional parts; a jetting zone for the jet-breakup of the core fluid and a UV exposure zone for photo-polymerization of the formed droplets. In the jetting zone, hydrophilic 
polyethyleneglycol diacrylate (PEGDA) is ejected from the central nozzle with shearing flows of hydrophobic hexadecane from both sides of the junction (Figure 1a and 1b). The capillary instability eventually ruptures the formed jet into uniform droplets (Figure 1c). A spiral channel with gradually increasing width maximizes UV exposure for the photo-polymerization of the prepolymer droplets while reducing coalescence (Figure 1d). ${ }^{33}$ The jet-mode requires short residence time and thus affords insufficient UV-exposure for the photo-polymerization, which is further challenged when large amount of QDs, fluorescent dyes, or the other light absorbing ingredients are loaded. The gradually increasing spiral channel provides continuous reduction of flow rate and correspondingly increased residence time to allow the photo-polymerization sufficient time. The diameter of the spiral channel was matched the circular UV beam from the UV microscope objective to achieve maximum exposure throughout the whole channel (left of Figure 1d).

\subsection{Characterization of the high-throughput jet-breakup}

High-speed imaging enables visualization and characterization of the jet-breakup process. In the channel (width $d_{c}$ ), a smooth jet of length $L_{j}$ and diameter $d_{j}$ forms at the nozzle (diameter $d_{n}$ ) as shown in Figure 2a. Along the jet-thread, the capillary instability induces a sinusoidal disturbance that grows and eventually causes the jet to break into a train of emulsion droplets with diameter $d_{e}$ (see also the high speed movie clip, Video S1, in the Supporting Information). ${ }^{25-27}$ Dual laser imaging provides further in-depth characterization of the jetbreakup phenomena (Figure 2b).

Double exposure images were made by twin Nd:YAG laser $(532 \mathrm{~nm}, 10 \mathrm{~Hz}, 7 \mathrm{~ns}$ pulse duration) with 500-1000 $\mu$ s interval. At outer and inner flow rates of $Q_{o u t}=20 \mu \mathrm{L} \mathrm{min}^{-1}$ and $Q_{i n}$ $=0.5 \mu \mathrm{L} \mathrm{min}{ }^{-1}$, respectively, the droplet speed is measured as $9.68 \mathrm{~cm} \mathrm{~s}^{-1}$ from the temporal 
phase shift, $\varphi_{e}$, in Figure 2b. The spatial wavelength of the emulsion droplets, $\lambda_{e}$, corresponds to a production rate of $381 \mathrm{~Hz}$ and generation tempo of $2.626 \mathrm{~ms}$. A maximum production rate of $2,320 \mathrm{~Hz}$ was achieved at $Q_{\text {out }}=30 \mu \mathrm{L} \mathrm{min}^{-1}$ and $Q_{\text {in }}=0.5 \mu \mathrm{L} \mathrm{min}{ }^{-1}$ with an emulsion diameter of $13 \mu \mathrm{m}$. The temporal phase shift and spatial wavelength of the jet instability also provide information about the characteristic time scale for the development of instabilities, fluctuations of the jet length, and ultimately the jet breakup.

At the junction area, a tapered geometry with a $15^{\circ}$ angle helps create stable shearing of the core prepolymer flow with straight and smooth features as shown in Figure 2c. (See also Figure S1) In the jet formation, it is important to produce an isolated jet confined in 3D. Wall contact would otherwise cause unstable flows with a resulting poor size distribution. Even though the jet starts from high aspect-ratio rectangular shaped nozzles (width: $20-40 \mu \mathrm{m}$, height: $100-160 \mu \mathrm{m})$, the interfacial tension drives the formation of an axisymmetric cylindrical jet confined by surrounding hexadecane flow. The cross-sectional views in Figure $2 \mathrm{~d}$ illustrate the development of the jet.

The jet-mode behavior can be characterized in terms of the Weber number of inner flow, $W e_{i n}$, and the capillary number of outer flow, $\mathrm{Ca}_{\text {out }}{ }^{25-30} \mathrm{We}$ is a measure of the relative importance of the fluid's inertia compared to its surface tension, and it is defined as $W e=\rho v^{2} L \gamma^{-1}$, where $\rho$ is density, $v$ the average velocity, $L$ is the nozzle diameter $\left(d_{n}\right)$, and $\gamma$ is the interfacial tension. $\mathrm{Ca}$ represents the relative effect of viscous forces versus surface tension and a defined as $C a=\mu v \gamma^{-1}$, where $\mu$ is the fluid viscosity. With increasing $W e_{i n}$ and $C a_{o u t}$, the mode transformed from dripping to jetting (Figure 3a). The droplet size expands with the inner flow rate (larger $W e_{i n}$ ). As the size of the droplet reaches the channel walls, the droplets slow down and begin to coalescence with each other. As we investigated the influence of the flow mode on 
the particle size and production rate, jet-mode breakup exhibited a much higher production rate than the dripping-mode with much smaller particle size. (See Figure S2) As the mode changed from jetting to dripping under this specific condition, the jet-mode production rate was 208 times higher than that achieved in dripping mode with $1 / 6$ of diameter. Here, $Q_{i n}$ was fixed at $0.5 \mu \mathrm{L}$ $\min ^{-1}$ while $Q_{o u t}$ was suddenly decreased from $30 \mu \mathrm{L} \min ^{-1}$ to $3 \mu \mathrm{L} \mathrm{min}{ }^{-1}$.

The jet-mode breakup also offers advantages compared to the dripping mode in control of particle size range (See also Figure S3). In the jet-mode, the particle diameter is determined by various parameters including nozzle size and the dimensionless numbers; $\mathrm{We}, \mathrm{Ca} .{ }^{34}$ Whereas in the dripping mode, the drop size is mostly dependent on the interfacial properties and the nozzle diameter. ${ }^{32}$ The size of the emulsions in jet-mode could be varied from $13 \mu \mathrm{m}$ to over $100 \mu \mathrm{m}$ over the flow rate range, but it ranged only from $52 \mu \mathrm{m}$ to $81 \mu \mathrm{m}$ in dripping mode (See Figure S3).

For an undisturbed jet, the ratio of emulsion size and jet diameter $d_{e} / d_{j}$ falls within the range $1.87-1.91 .^{25,28,30}$ These values can be achieved only under a stable laminar flow condition. ${ }^{18}$ This flow condition is difficult to achieve in conventional dispensing systems since most are operated under turbulent conditions. ${ }^{35}$ On the other hand, a microfluidic system provides an ideal low Reynolds number environment with its small dimensions and precisely crafted geometry. The measured reduced diameters from our microfluidic system are in the expected range (Figure 3b) over a wide range of flow rates indicating a broad operating window of stable jet behavior. As a result, produced emulsion shows uniform size distribution as shown in Fig. $3 \mathrm{c}$ and $3 \mathrm{~d}$. The average diameter is $31 \mu \mathrm{m}$ with $3 \%( \pm 1 \mu \mathrm{m})$ standard deviation. This narrow size distribution is comparable to the excellent uniformity characteristic of dripping mode while the production rate is two orders of magnitude higher at same flow rate. 


\subsection{Preparation of the quantum dot embedded microbeads}

Uniform PEGDA emulsions made by jet-breakup were photochemically polymerized in the UV exposure zone with spiral channel. As an example, Figure 4a shows an optical micrograph of highly uniform emulsions flowing along the spiral channel without any stability issues (see also the high speed movie clip, Video S2, in the Supporting Information). The polymerized PEGDA particles were directly collected from the device outlet (Figure 4b). The scanning electron microscopy (SEM) image in Figure 4c confirms the uniformity and welldefined globular shape of the crosslinked PEGDA particles.

QD-doped microbeads have received considerable attention due to their useful optical properties for many potential applications. However, most of the developed incorporation methods are based on low yield batch process. For example, Han et al. reported diffusive transport of QDs from the solvent phase into the dispersed polymer beads ${ }^{7}$ and Insin et al. presented deposition of the QDs on the microbeads to prepare QD-doped particles. ${ }^{10}$ Since the diffusive transport of nanoparticles in a viscous prepolymer resin is slow, the diffusion based QD-embedding process requires long processing time with large amount of materials. Moreover, the efficiency decreases as the size of loading material becomes larger. On the other hand, deposition method allows adsorption of QDs merely on the particle surface, making it hard to create highly loaded particles even with multistep layer-by-layer deposition process. Moreover, in both cases, a significant portion of loading materials is wasted during the multistep process of synthesis, purification, loading, and washing. ${ }^{7,10}$ This is especially not desirable when expensive loading materials, such as QDs, are used.

In this study, we synthesized CdSe/ZnS coreshell QDs (504 nm and $560 \mathrm{~nm}$ emission) by continuous and batch processes to incorporate into the polymer beads. ${ }^{36,37}$ Especially, large QDs 
(12 $\mathrm{nm}$ diameter) with thick $\mathrm{ZnS}$ shells were prepared and used to reduce oxidation and luminescence quenching in the polymer (See Figure S4 and S5). Such a particle with large diameter is hard to incorporate into the microparticles through diffusion process. An advantage of using this microfluidic jetting platform is the versatility of material loading within the biocompatible polymer particles. It is easy to put any combination of hydrophilic ingredient into the PEGDA monomer by simply mixing them together prior to injection, but hydrophobic materials can also be incorporated into the PEGDA after surface modification with PEG-based ligands.

Fluorescent images (Figure 4d) show uniform QD-doped fluorescent particles with 9.9 $\mathrm{wt} \%$ of loading and $504 \mathrm{~nm}$ of peak emission. In general, prepolymer resin with highly loaded QDs shows significant red-shift, peak-broadening, distortion or secondary peak formation caused by agglomeration of colloidal QD nanoparticles. (See Figure S5) In this study, surface of the QDs were modified with PEG-thiols compatible and dispersible with PEGDA prepolymer. Additionally, CdSe nanocrystals coated with thick $\mathrm{ZnS}$ shells were introduced to prevent aforementioned issue by confining electrons even with high QD loading. A comparison of photoluminescence from QDs dispersed in chloroform, prepolymer resin and crosslinked PEGDA (Figure 4e) shows that the QD optical properties remain essentially unchanged even at high concentration of QDs, only $1.3 \mathrm{~nm}$ red-shift of peak emission and $0.62 \mathrm{~nm}$ difference of FWHM is observed (See Figure S5 for the other QDs).

We measured the solid contents of the QD solution two different ways; optical absorbance and density measurement. ${ }^{38}$ From the location of the first absorption peak and particle diameter, we estimated number of cores and their volumetric percentage. However, this calculation method consistently showed much higher QD loadings than the density based 
estimates because it assumes highly diluted concentration of QDs. Since very high concentration of QD solutions with significant self-absorption were used, we report only values based on the density measurement. Solid contents of the particles used in the microfluidics experiments were ranged from $2.2-3.5 \mathrm{vol} \%(7.1-11.9 \mathrm{wt} \%)$. To our knowledge, this is the largest value reported for QD loaded polymer particles to date. Beyond 5.6 vol\%, viscosity of the loaded prepolymer resin increased significantly and the resin completely gelled above 9 vol\% (20 wt $\%)$. Compared with diluted resin, these thick resins show significant self-absorption. (See Figure S4)

\section{Conclusions}

In conclusion, we have realized coherent jet-mode followed by stable drop breakup in a microfluidic system with a nozzle designed for the rapid production of biocompatible prepolymer emulsions loaded with fluorescent QDs. A spiraling and gradually widening channel enabled maximum absorption of radiated UV light for the in-line photopolymerization without coalescence and clogging issues. Compared to the often used dripping mode, the developed microfluidic jet-breakup scheme provided advantages in an expanded range operating conditions from $13 \mu \mathrm{m}$ to $110 \mu \mathrm{m}$ with over 38 times higher productivity at $2.32 \mathrm{kHz}$ while still retaining a narrow size distribution of $3 \%$. By using large CdSe/ZnS QDs with PEG-terminated ligand well dispersible in PEGDA, uniform biocompatible microparticles with more than $10 \mathrm{wt} \%$ of very high QD loadings were achieved. The final QD load polymer particles had a $28 \mathrm{~nm}$ of narrow emission band width at $504 \mathrm{~nm}$ and $560 \mathrm{~nm}$ without significant peak broadening or distortion. 
a)

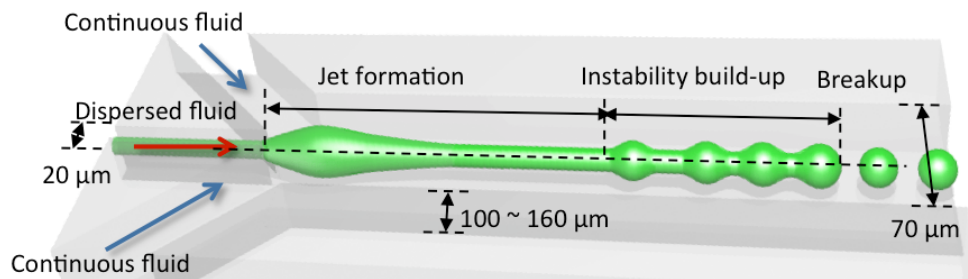

Continuous fluid
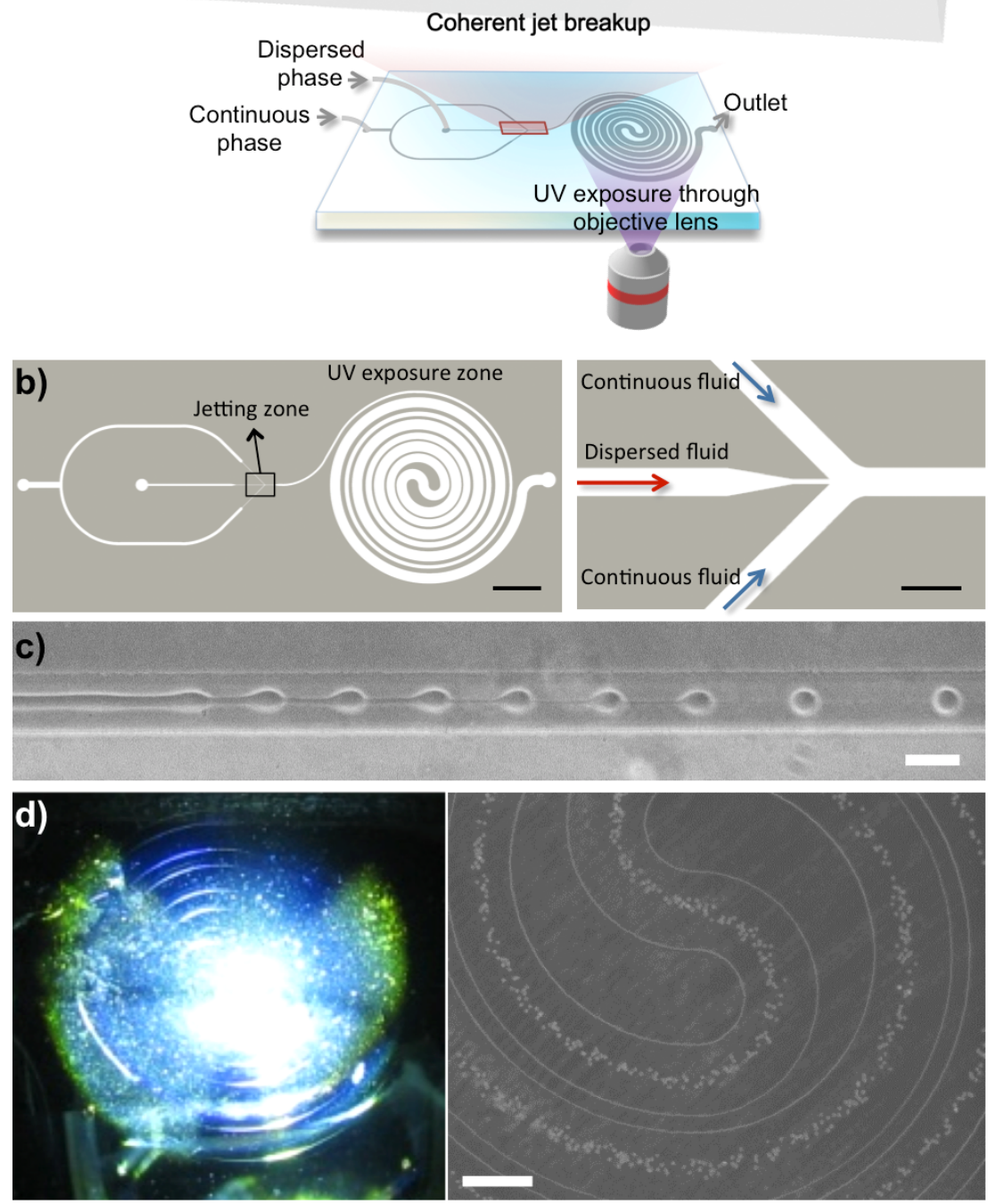

Figure 1. Gallery of the high performance microparticle generator. The design is optimized for coherent and high rate jet-breakup and subsequent photo-polymerization to produce uniform fluorescent microbeads in continuous manner. (a) Schematic of the jetting zone with $40 \times 100 \mu \mathrm{m}$ nozzle in the center for hydrophilic PEGDA ejection. Hexadecane is introduced through the cross channels. (b) Overall chip design (left, scale bar $=3 \mathrm{~mm}$ ) and nozzle design (right, scale bar $=200 \mu \mathrm{m}$ ) from CAD drawing. (c) Optical micrograph of jet-breakup corresponds to the 
schematic in (a). Scale bar is $100 \mu \mathrm{m}$. (d) UV exposure zone with gradually widening spiral flow channel for the in situ polymerization of PEGDA. Left image shows spot of UV exposure on the overall spiral channel. Scale bar is $1000 \mu \mathrm{m}$. Right image shows highly uniform emulsions flowing along the spiral channel without any stability issues (at $Q_{o u t}=50 \mu \mathrm{L} \mathrm{min}^{-1}, Q_{\text {in }}=0.5 \mu \mathrm{L}$ $\left.\min ^{-1}\right)$. 

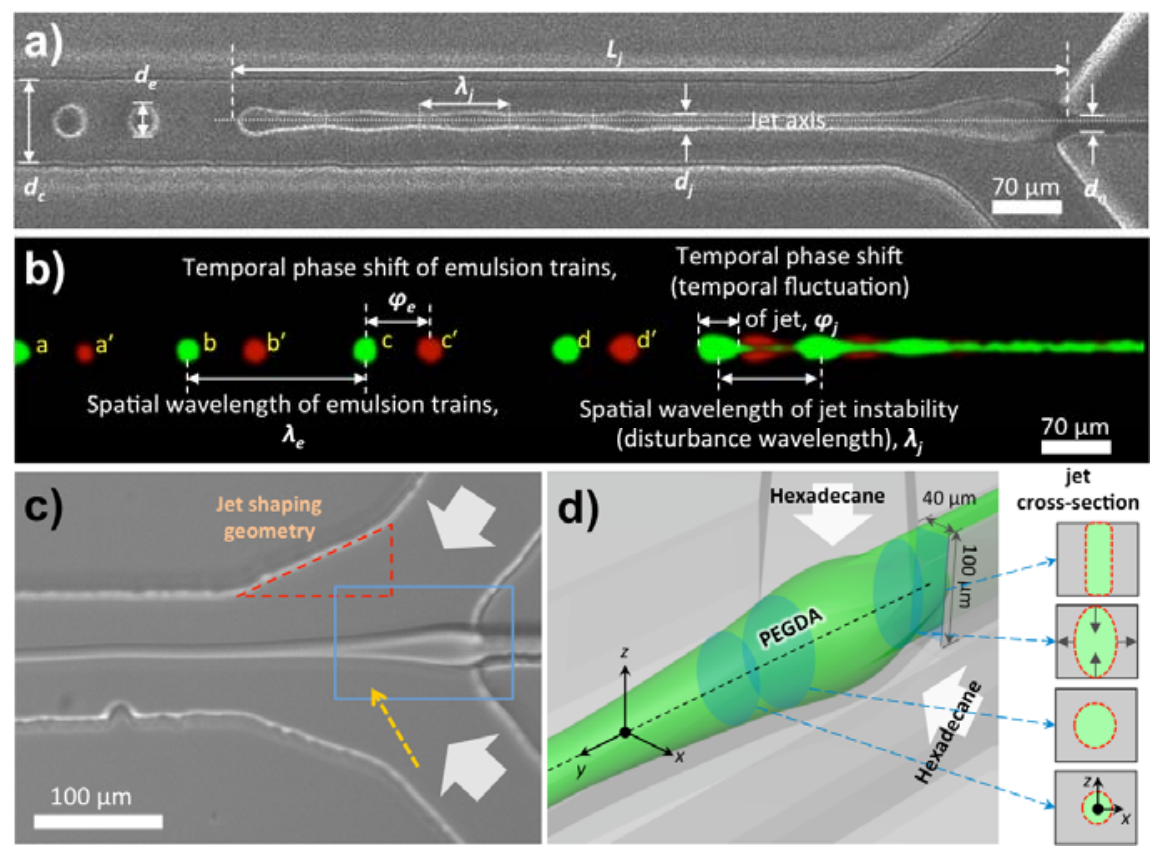

Figure 2. High-speed images and dual-pulsed laser photography images for the characterization of the jet-mode at $Q_{o u t}=20 \mu \mathrm{L} \mathrm{min}{ }^{-1}, Q_{i n}=0.5 \mu \mathrm{L} \mathrm{min} \operatorname{mos}^{-1}$. (a) High-speed image showing characteristic features of jet-mode breakup mechanism as a series of events; ejection, elongation, instability build-up and breakup. Jet parameters are emulsions diameter, $d_{e}$, jet diameter, $d_{j}$, channel diameter, $d_{c}$, nozzle diameter $d_{n}$, disturbance wavelength, $\lambda_{j}$ and jet length, $L_{j}$. (b) Dualpulsed laser photography provides information about the spatial interval $\left(\lambda_{e}, \lambda_{j}\right)$ and temporal shift $\left(\varphi_{e}, \varphi_{j}\right)$ of emulsion trains and jet (red first image, green second image). (c) Magnified image of nozzle area designed for the shaping of a stable jet. By shaping the edge of crosschannel (red triangle), the shearing flow gets smaller adjacent angle improving the stability of the jet. (d) Schematic corresponding to the blue box of (c) showing how the jet is formed and remain isolated from channel walls. 

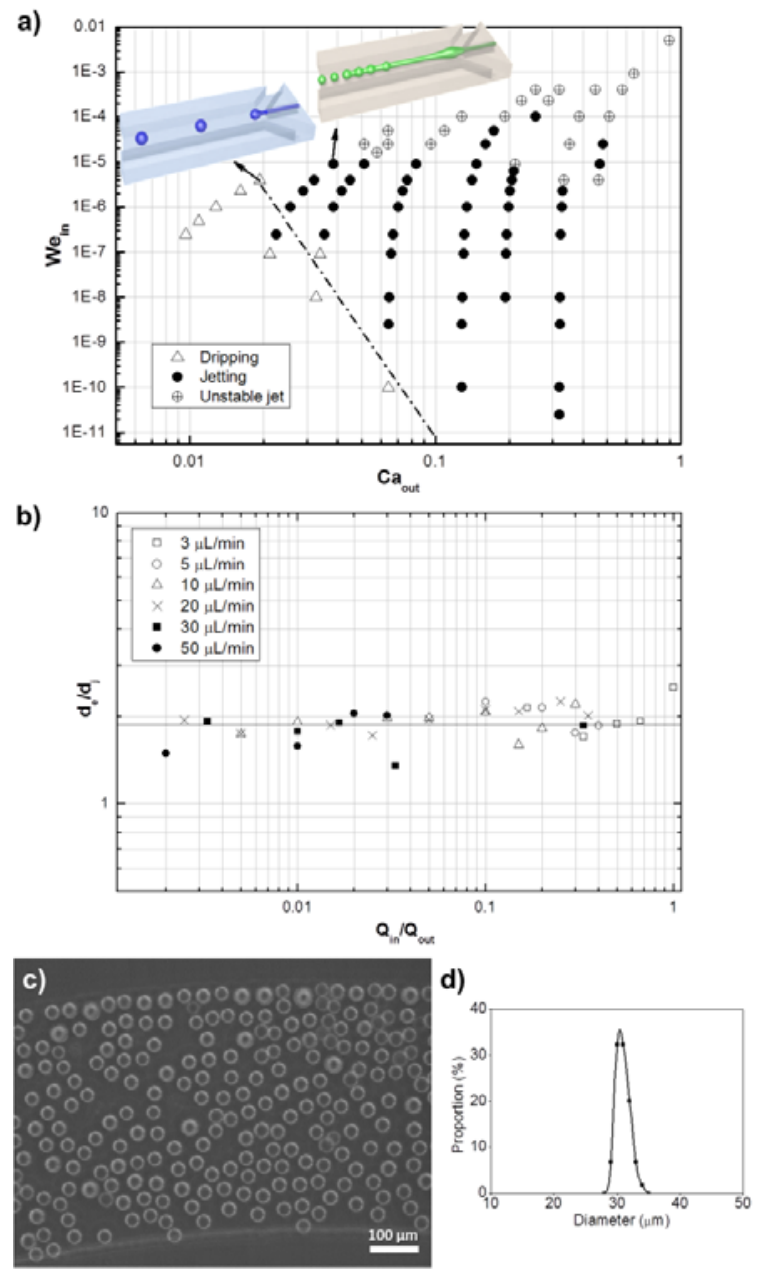

Figure 3. Behavior of jet with fluid parameters. (a) Flow phase diagram plotted with $W e_{\text {in }}$ versus Ca $a_{\text {out. }}$ At $0.01-0.1$ of $C a_{\text {out }}$, the flow transitions from dripping (triangle) to jetting (black dot). The jet-mode region expands with increasing $W e_{\text {in }}$, but above a certain value of $W e_{\text {in }}\left(\sim 10^{-5}\right)$, an unstable breakup pattern (crossed circle) appears. (b) Plot of reduced dimensionless diameters $\left(d_{e} / d_{j}\right)$ over the various relative volumetric flow rates $\left(Q_{i n} / Q_{o u t}\right)$. The data converge around the expected value from theoretical studies, 1.87, indicating that the jet-mode is stable over a wide range of flow conditions. (c) Optical micrographs of highly uniform emulsions flowing along the

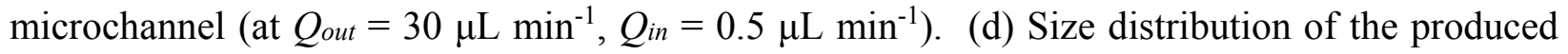
microparticles as narrow as $3 \%(31 \pm 1 \mu \mathrm{m})$. 


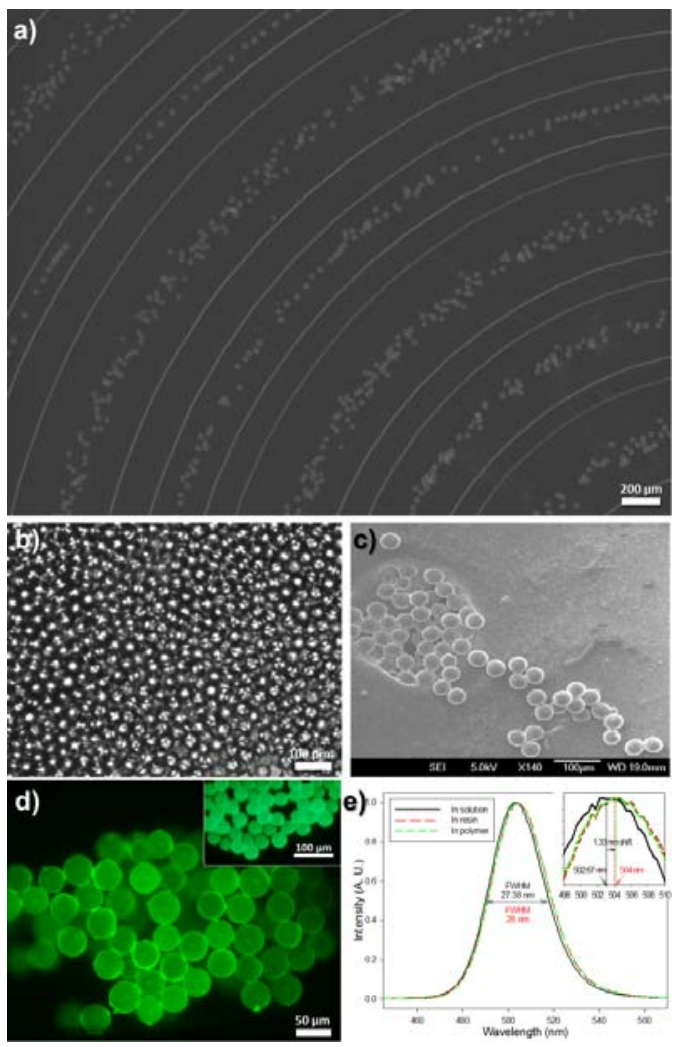

Figure 4. Images and characteristics of the polymerized microparticles. (a) Uniform $24 \mu \mathrm{m}$ PEGDA particles flowing along the spiral channel. (at $Q_{o u t}=50 \mu \mathrm{L} \min ^{-1}, Q_{i n}=0.5 \mu \mathrm{L} \min ^{-1}$ ) (b) Transmission optical micrograph of the uniform PEGDA microbeads. (c) SEM image of PEGDA microbeads verifying highly uniform sizes. (d) Pseudo-color fluorescent image of highly monodisperse PEGDA polymer beads with $9.9 \mathrm{wt} \%$ of highly loaded CdSe/ZnS coreshell nanocrystals. The inset shows a real color image from the same QD-doped particles. (e) Photoluminescence spectra from QDs which are dispersed in chloroform (black), PEGDA monomer (red) and polymerized PEGDA (green), respectively. The QD loaded polymer beads have strong emission at $504 \mathrm{~nm}$ with $28 \mathrm{~nm}$ narrow FWHM (full-width half-maximum). The insert shows that only a small $1.3 \mathrm{~nm}$ red-shift and $2 \%$ widening in FWHM in the solvent 
exchange from chloroform to PEGDA. Moreover, no shift or widening of the peak is observed during photo-polymerization.

\section{AUTHOR INFORMATION}

\section{Corresponding Author}

*Department of Chemical Engineering. Massachusetts Institute of Technology, 77 Massachusetts Avenue, 66-342, Cambridge, MA 02139 (USA), Fax: +1-617-258-8992; Tel: +1-617-253-4589; E-mail: kfjensen@mit.edu

\section{Author Contributions}

The manuscript was written through contributions of all authors. All authors have given approval to the final version of the manuscript.

\section{ACKNOWLEDGMENT}

The authors thank the US Army Research Office through the Institute for Soldier Nanotechnology (DAAD-19-02-0002) for support. We thank Dr. S.-H. Kim at KAIST for the tensiometry experiments. SKL would like to dedicate this work to Prof. Seung-Man Yang, a great scientist and a mentor, who passed away on September 26th, 2013.

\section{SUPPORTINg INFORMATION}

Supporting Information and video clips are available free of charge via the Internet at http://pubs.acs.org. 


\section{REFERENCES}

(1) Gao, X.; Cui, Y.; Levenson, R. M.; Chung, L.W.K.; Nie, S. In vivo cancer targeting and imaging with semiconductor quantum dots. Nat. Biotechnol. 2004, 22, 969-976.

(2) Tang, B. C.; Dawson, M.; Lai, S. K.; Wang, Y. -Y.; Suk, J. S.; Yang, M.; Zeitlin, P.; Boyle, M. P.; Fu, J.; Hanes, J. Biodegradable polymer nanoparticles that rapidly penetrate the human mucus barrier. Proc. Natl. Acad. Sci. U. S. A. 2009, 106, 19268-19273.

(3) De Geest, B. G.; Urbanski, J. P.; Thorsen, T.; Demeester, J.; De Smedt, S. C.; Monodisperse microgel synthesis inside microfluidic devices. Langmuir 2005, 21, 10275-10279.

(4) Kim, S. -H.; Lim, J. -M.; Lee, S. -K.; Heo, C. -J.; Yang, S. -M. Biofunctional colloids and their assemblies. Soft Matter 2010, 6, 1092-1110.

(5) Pregibon, D. C.; Toner, M.; Doyle, P. S. Multifunctional encoded particles for highthroughput biomolecule analysis. Science 2007, 315, 1393-1396.

(6) Braeckmans, K.; De Smedt, S. C.; Roelant, C.; Leblans, M.; Pauwels, R.; Demeester, J. Encoding microcarriers by spatial selective photobleaching. Nat. Mater. 2003, 2, 169-173.

(7) Han, M.; Gao, X.; Su, J. Z.; Nie, S. Quantum-dot-tagged microbeads for multiplexed optical coding of biomolecules. Nat. Biotechnol. 2001, 19, 631-635.

(8) Snee, P. T.; Chan, Y.; Nocera, D. G.; Bawendi, M. G. Whispering-gallery-mode lasing from a semiconductor nanocrystal/microsphere resonator composite. Adv. Mater. 2005, 17, 1131-1136. 
(9) Kim, S. -H.; Jeon, S. -J.; Yi, G. -R.; Heo, C. -J.; Choi, J. H.; Yang, S. -M. Optofluidic assembly of colloidal photonic crystals with controlled sizes, shapes, and structures. Adv. Mater. 2008, 20, 1649-1655.

(10) Insin, N.; Tracy, J. B.; Lee, H.; Zimmer, J. P.; Westervelt, R. M.; Bawendi, M. G. Incorporation of iron oxide nanoparticles and quantum dots into silica microspheres. ACS Nano, 2008, 2, 197-202.

(11) Xu, S.; Nie, Z.; Seo, M.; Lewis, P.; Kumacheva, E.; Stone, H. A.; Garstecki, P.; Weibel, D. B.; Gitlin, I.; Whitesides G. M. Generation of monodisperse particles by using microfluidics: control over size, shape, and composition. Angew. Chemie. Int. Ed. 2005, 44, 724-728.

(12) Kim, J. -W.; Utada, A. S.; Fernández-Nieves, A.; Hu, Z.; Weitz, D. A. Fabrication of monodisperse gel shells and functional microgels in microfluidic devices. Angew. Chemie. Int. Ed. 2007, 46, 1819-1822.

(13) Choi, C.-H.; Jung, J.-H.; Hwang, T.-S.; Lee, C.-S. In situ microfluidic synthesis of monodisperse PEG microspheres. Macromol. Res. 2009, 17, 163-167.;

(14) Zhao, Y.; Shum, H. C.; Chen, H.; Adams, L. L. A.; Gu, Z.; Weitz, D. A. Microfluidic generation of multifunctional quantum dot barcode particles. J. Am. Chem. Soc. 2011, 133, 87908793.

(15) Wan, J. Microfluidic-based synthesis of hydrogel particles for cell microencapsulation and cell-based drug delivery. Polymers 2012, 4, 1084-1108.

(16) Thorsen, T.; Roberts, R. W.; Arnold, F. H.; Quake. S. R. Dynamic pattern formation in a vesicle-generating microfluidic device. Phys. Rev. Lett. 2001, 86, 4163-4166. 
(17) Kim, J. -Y.; deMello, A. J.; Chang, S. -I.; Hong, J.; O’Hare, D. Thermoset polyester droplet-based microfluidic devices for high frequency generation. Lab Chip, 2011, 11, 41084112.

(18) Agresti, J. J.; Antipov, E.; Abate, A. R.; Ahn, K.; Rowat, A. C.; Baret, J. -C.; Marquez, M.; Klibanov, A. M.; Griffiths, A. D.; Weitz, D. A. Ultrahigh-throughput screening in dropbased microfluidics for directed evolution. Proc. Natl. Acad. Sci. U. S. A. 2010, 107, 4004-4009.

(19) Anna, S. L.; Bontoux, N.; Stone, H. A. Formation of dispersions using 'flow-focusing' in microchannels. Appl. Phys. Lett. 2003, 82, 364-366.

(20) Holtze, C.; Rowat, A. C.; Agresti, J. J.; Hutchison, J. B.; Angilè, F. E.; Schmitz, C. H. J.; Köster, S.; Duan, H.; Humphry, K. J.; Scanga, R. A.; Johnson, J. S.; Pisignano, D.; Weitz, D. A. Biocompatible surfactants for water-in-fluorocarbon emulsions. Lab Chip 2008, 8, 1632-1639.

(21) Abate, A. R.; Weitz, D. A. Air-bubble-triggered drop formation in microfluidics. Lab Chip 2011, 11, 1713-1716.

(22) Chen, C. -H.; Abate, A. R.; Lee, D.; Terentjev, E. M.; Weitz, D. A. Microfluidic assembly of magnetic hydrogel particles with uniformly anisotropic structure. Adv. Mater. 2009, 21, 32013204.

(23) Dendukuri, D., Tsoi, K., Hatton, T.A., Doyle, P. S. Controlled synthesis of non-spherical microparticles using microfluidics. Langmuir 2005, 21, 2113-2116.

(24) Kim, S. -H.; Jeon, S. -J.; Yang, S. -M. Optofluidic encapsulation of crystalline colloidal arrays into spherical membrane. J. Am. Chem. Soc. 2008, 130, 6040-6046.

(25) Strutt, J. W. On the instability of jets. Proc. London Math. Soc. 1878, 10, 4-13. 
(26) Middleman, S. Modeling axisymmetric flows: dynamics of films, jets, and drops; Academic Press: San Diego, CA, 1995.

(27) de Gennes, P.-G.; Brochard-Wyart, F.; Quéré, D. Capillary and wetting phenomena-drops, bubbles, pearls, waves; Springer: New York, 2002.

(28) Teng, H.; Kinoshita, C. M.; Masutani, S. M. Prediction of droplet size from the breakup of cylindrical liquid jets. Int. J. Multiphase Flow 1995, 21, 129-136.

(29) Park, J. I.; Saffari, A.; Kumar, S.; Gunther, A.; Kumacheva, E. Microfluidic synthesis of polymer and inorganic particulate materials. Annu. Rev. Mater. Res. 2010, 40, 415-443.

(30) Utada, A. S.; Fernández-Nieves, A.; Stone, H. A.; Weitz, D. A. Dripping to jetting transitions in coflowing liquid streams. Phys. Rev. Lett. 2007, 99, 094502.

(31) Brouzes, E.; Medkova, M.; Savenelli, N.; Marran, D.; Twardowski, M.; Hutchison, J. B.; Rothberg, J. M.; Link, D. R.; Perrimon, N.; Samuels, M. L. Droplet microfluidic technology for single-cell high-throughput screening. Proc. Natl. Acad. Sci. U. S. A. 2009, 106, 14195-14200.

(32) Günther, A.; Jhunjhunwala, M.; Thalmann, M.; Schmidt, M. A.; Jensen, K. F. Micromixing of miscible liquids in segmented gas-liquid flow. Langmuir 2005, 21, 1547-1555.

(33) Hartman, R. L.; Naber, J. R.; Zaborenko, N.; Buchwald, S. L.; Jensen, K. F. Overcoming the challenges of solid bridging and constriction during Pd-catalyzed $\mathrm{C}-\mathrm{N}$ bond formation in microreactors. Org. Process Res. Dev. 2010, 14, 1347-1357.

(34) Utada, A.S.; Fernández-Nieves, A.; Gordillo, J. M.; Weitz, D. A. Absolute instability of a liquid jet in a coflowing stream. Phys. Rev. Lett. 2008, 100, 014502. 
(35) Schramm, L. L. Emulsions, Foams, and Suspensions: Fundamentals and Applications; Wiley-VCH: Weinheim, Germany, 2005.

(36) Marre, S.; Park, J.; Rempel, J.; Guan, J.; Bawendi, M. G.; Jensen, K. F. Supercritical continuous-microflow synthesis of narrow size distribution quantum dots. Adv. Mater. 2008, 20, $4830-4834$.

(37) Dabbousi, B. O.; Rodriguez-Viejo, J.; Mikulec, F. V.; Heine, J. R.; Mattoussi, H.; Ober, R.; Jensen, K. F.; Bawendi, M. G. (CdSe)ZnS Core-Shell Quantum Dots: Synthesis and characterization of a size series of highly luminescent nanocrystallites. J. Phys. Chem. B 1997, $101,9463-9475$.

(38) Yu, W. W.; Qu, L.; Guo, W.; Peng, X. Experimental determination of the extinction coefficient of CdTe, CdSe, and CdS nanocrystals. Chem. Mater. 2003, 15, 2854-2860. 


\title{
High Throughput Synthesis of Uniform Biocompatible Polymer Beads with High Quantum Dot Loading Using Microfluidic Jet-Mode Breakup
}

\author{
by Seung-Kon Lee, Jinyoung Baek and Klavs F. Jensen* \\ E-mail: kfjensen@mit.edu
}

Table S1 Comparison of high throughput microfluidic emulsion production system

\begin{tabular}{|c|c|c|c|c|c|c|}
\hline Developer & $\begin{array}{l}\text { Device } \\
\text { material }\end{array}$ & $\begin{array}{l}\text { Maximum } \\
\text { production } \\
\text { rate }[\mathrm{kHz}]\end{array}$ & $\begin{array}{l}\text { Core flow rate at for } \\
\text { miximum production } \\
\text { rate }[\mu \mathrm{L} / \mathrm{min}]\end{array}$ & $\begin{array}{l}\text { Operation } \\
\text { mode }\end{array}$ & Uniformity & Production material \\
\hline $\begin{array}{l}\text { J.-Y. Kim et al. } \\
\text { [a] }\end{array}$ & $\begin{array}{l}\text { Thermoset } \\
\text { polyester }\end{array}$ & 1.73 & 20 & Dripping & Good & Water emulsion \\
\hline $\begin{array}{l}\text { J. J. Agrestia et } \\
\text { al. [b] }\end{array}$ & PDMS & 2 & 6.67 & Dripping & Good & Water emulsion \\
\hline $\begin{array}{l}\text { Shelley L. Anna } \\
\text { et al. [c] }\end{array}$ & PDMS & 10 & - & $\begin{array}{l}\text { Dripping } \\
\text { /Jetting }\end{array}$ & Varies [d] & Water emulsions \\
\hline $\begin{array}{l}\text { C. Holtze et al. } \\
\text { [e] }\end{array}$ & PDMS & 10 & 1.67 & Dripping & Good & Water emulsions \\
\hline $\begin{array}{l}\text { Adam R. Abate } \\
\text { et al. [f] }\end{array}$ & PDMS & 6 & 1.67 & Jetting [g] & Good & Water emulsions \\
\hline $\begin{array}{l}\text { S. -K Lee et } \\
\text { al.[h] }\end{array}$ & PDMS & 2.32 & 0.5 & Jetting & $3 \%$ & $\begin{array}{l}\text { PEGDA prepolymer } \\
\text { emulsion/ } \\
\text { Polymer particles }\end{array}$ \\
\hline
\end{tabular}

[a] Lab Chip, 2011, 11, 4108-4112.

[b] Proc. Nat. Acad. Sci. 2010, 107, 4004-4009.

[c] Appl. Phys. Lett. 2003, 82, 364-366.

[d] Varies according to the flow rate condition

[e] Lab Chip 2008, 8, 1632-1639.

[f] Lab Chip 2011, 11, 1713-1716.

[g] Bubble triggered jet-breakup

[h] Present work 
a

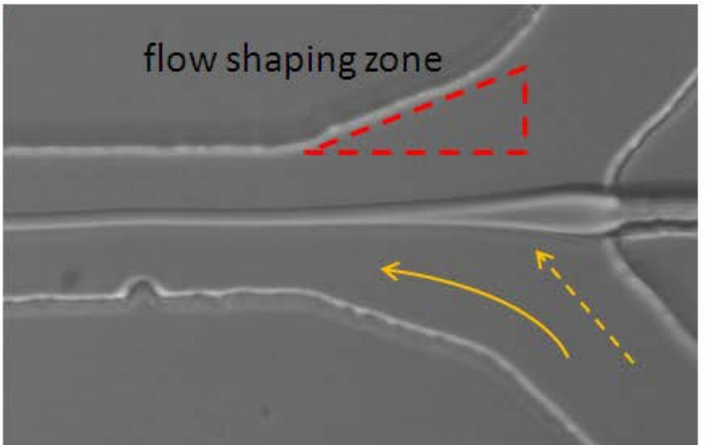

b

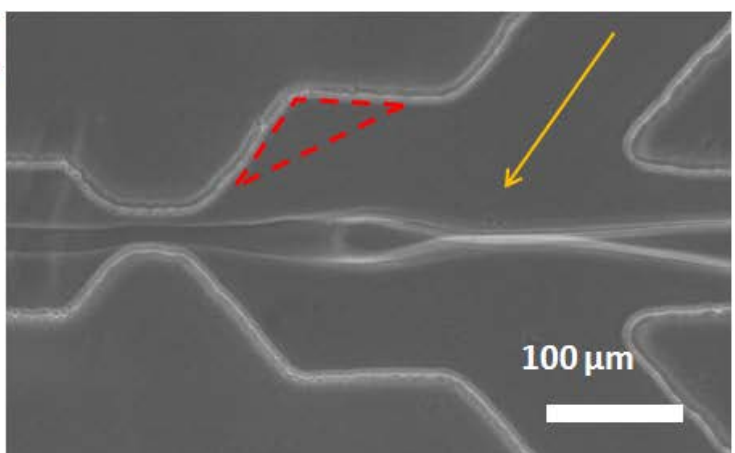

Figure S1 Effect of proper design for the formation of stable jet-flow. a) By applying a shaping zone around the nozzle, a more stable jet-stream is produced. b) Without the shaping zone, the side flows destabilize the jet.
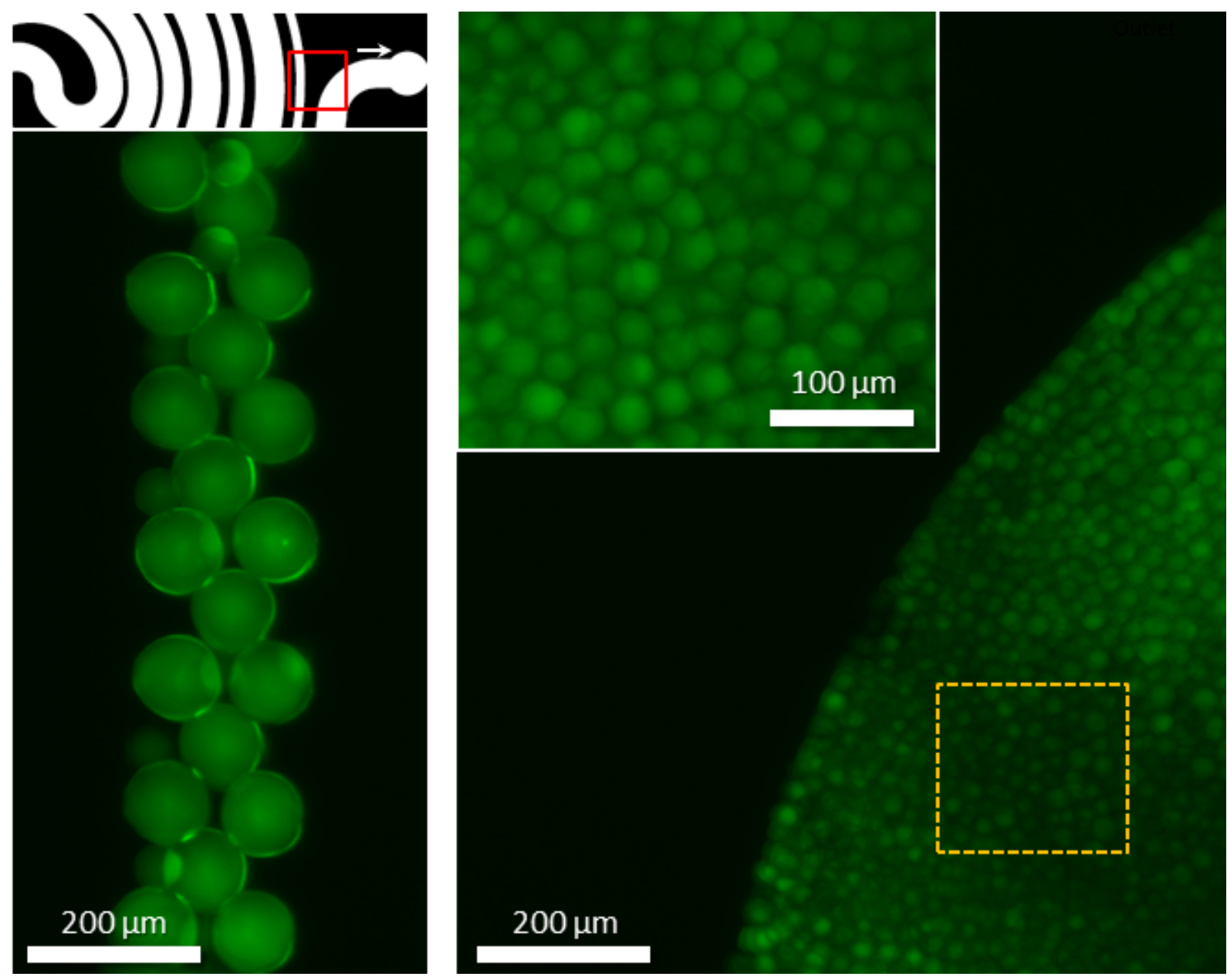

Figure S2 Fluorescent image of the crosslinked PEGDA microbeads encoded with QD nanoparticles taken during the phase transition from jetting (right side) to dripping (left side). Inset (left) shows the location of the image was taken. Magnified inset image (right) illustrates size and productivity of microbeads generated in jet-mode when compared with those produced in dripping mode (left). Scale bar is $100 \mu \mathrm{m}$. 


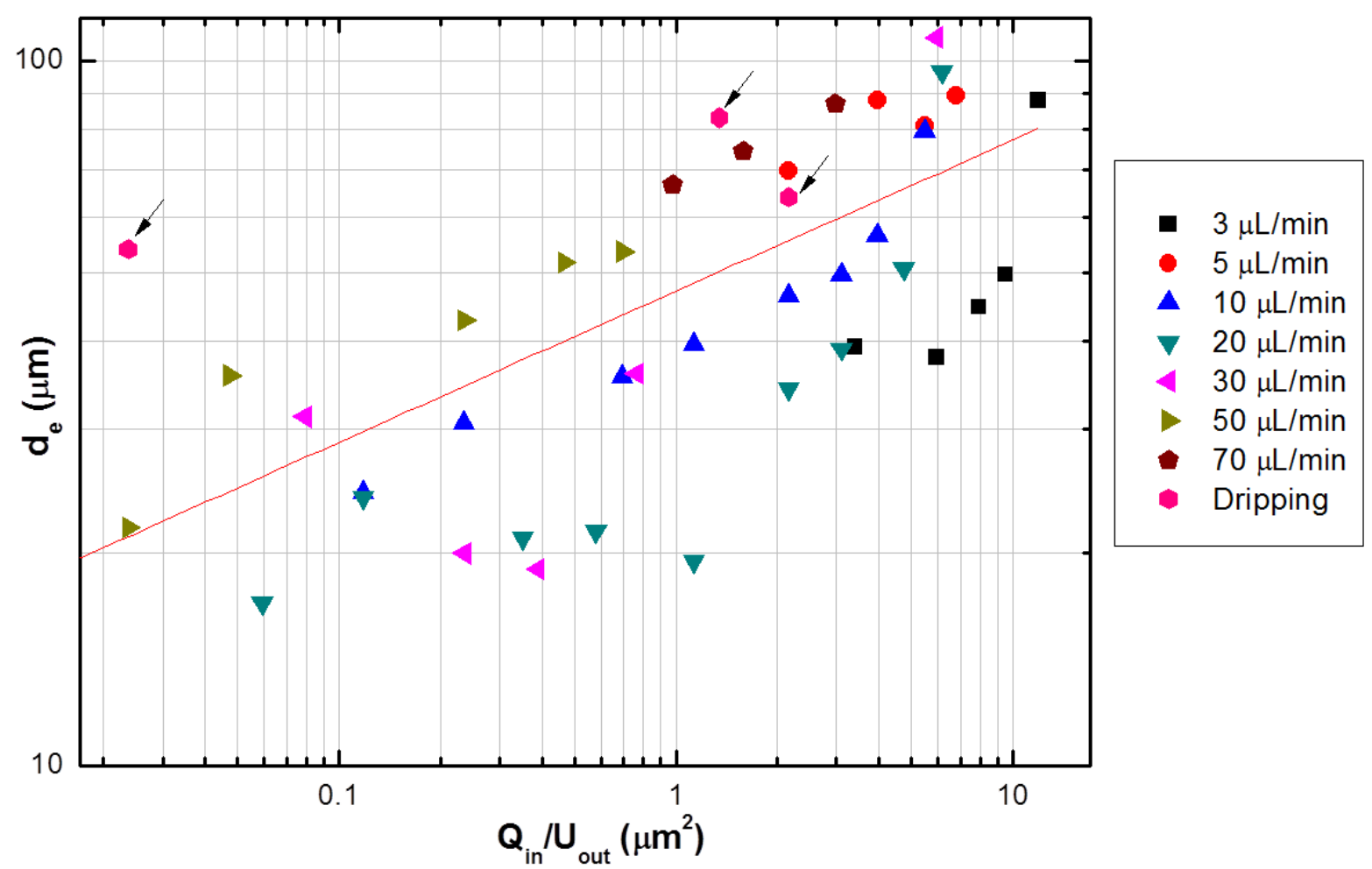

Figure S3 Variation of emulsion diameter with the relative flow rate change. The emulsion size can be controlled from $13 \mu \mathrm{m}$ to over $100 \mu \mathrm{m}$ of emulsion size. After polymerization, the smallest size becomes $10 \mu \mathrm{m}$ with a 20 30\% of shrinkage. The scatter is attributed to interactions with the wall. When the emulsion size (or jet diameter) is small, the disturbance is reduced. In dripping mode (magenta hexagons denoted with arrows), size of the produced droplets range from $52 \mu \mathrm{m}$ to $81 \mu \mathrm{m}$. The controllable range with jet-mode breakup is $\sim 3$ times wider than that of dripping mode. 


\section{a}

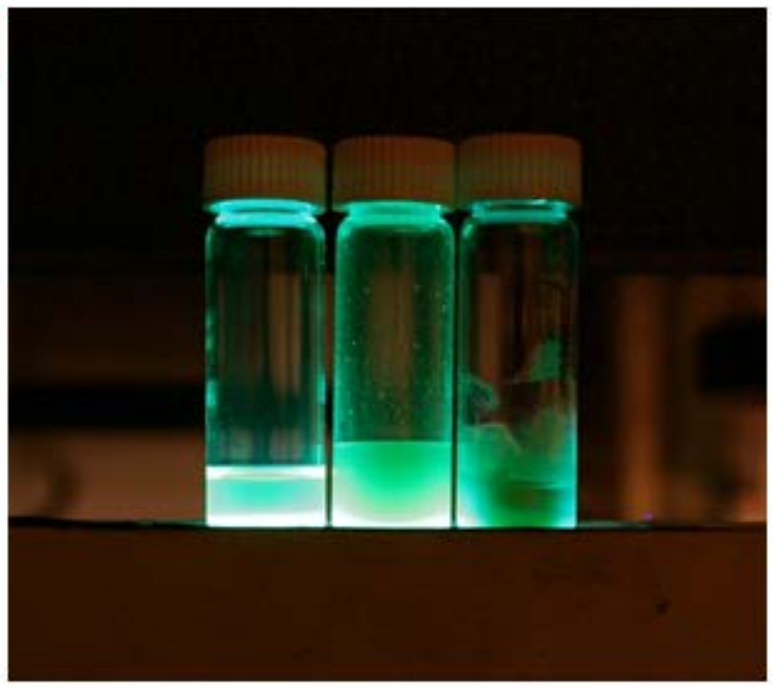

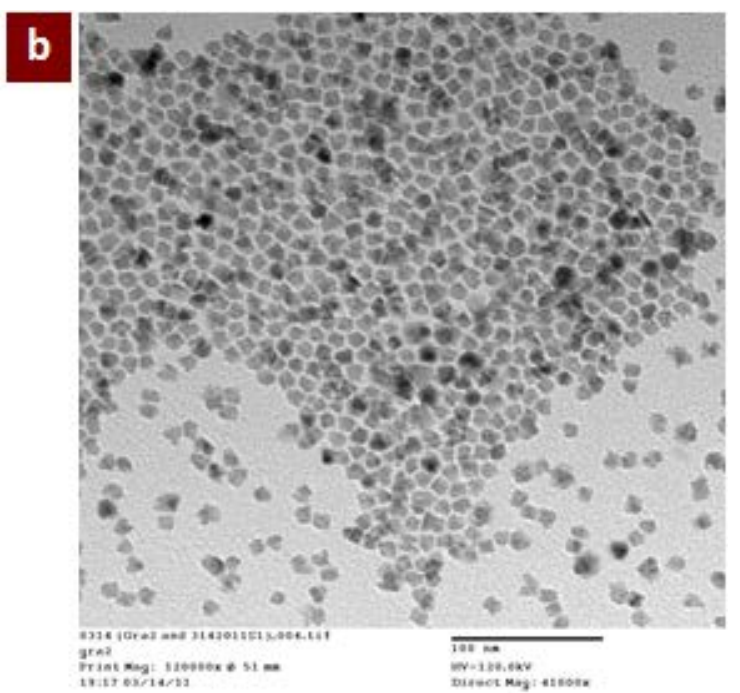

Figure S4 Photograph of QD loaded PEGDA prepolymer resin placed on the UV lamp and the TEM image of QDs. a) Fluorescent prepolymer resin doped with CdSe/ZnS QDs with $504 \mathrm{~nm}$ emission peak. From the left to right, concentration increased from 1, 3.5 to 9 vol\%. Over 5 vol\% (20 wt \%) the resin starts to gel because of colloidal crystallization driven by high inter particular interaction. The reduced emission with increasing loading results from self-absorption from highly concentrated QDs among the prepolymer resin. b) Transmission electron microscopy (TEM) image thick shell CdSe/ZnS showing uniform size with an average diameter of $12 \mathrm{~nm}$.
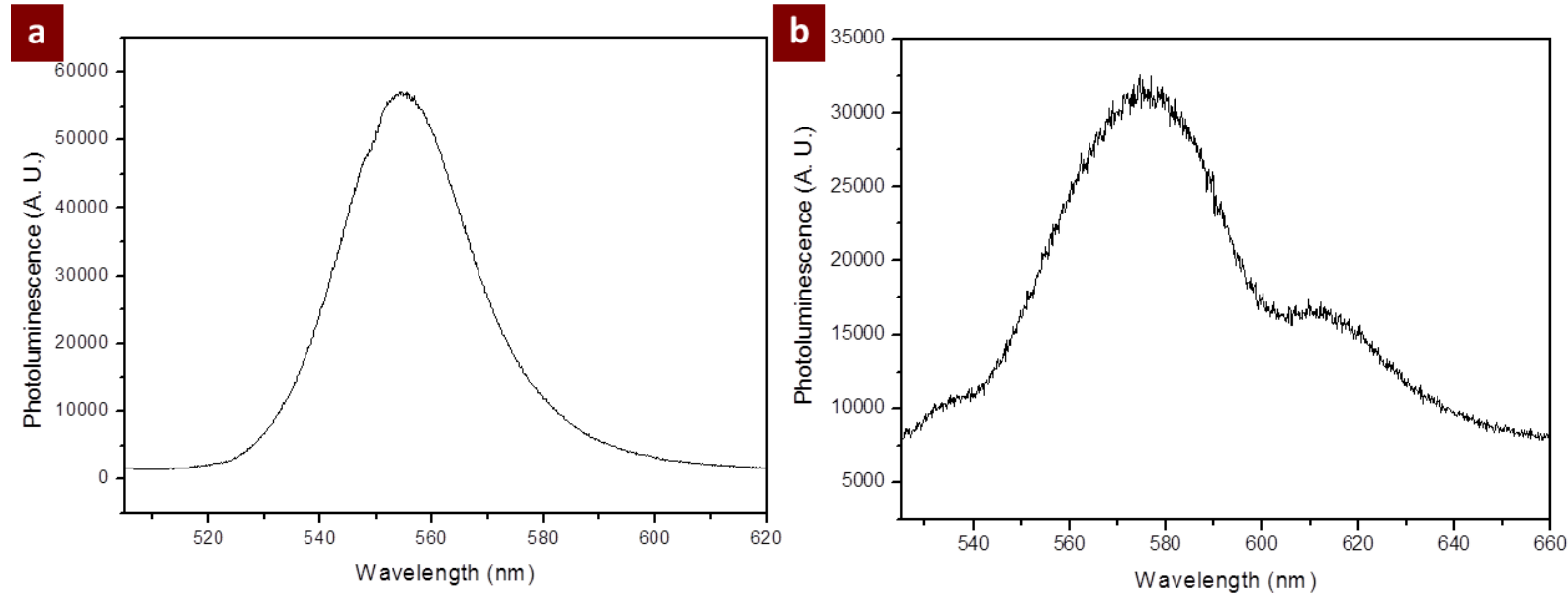

Figure S5 Photoluminescence spectra from QD-doped PEGDA polymers with and without thick protective shells and PEG-based surface modification. (a) CdSe/ZnS coreshell QDs with thick ZnS shells and PEG-thiol ligand. It shows smooth and narrow emission at $554.4 \mathrm{~nm}$ with a band width of $28 \mathrm{~nm}$. (b) CdSe/ZnS coreshell QDs with thin ZnS shells without surface modification. Due to the agglomeration and phase separation of QDs in PEGDA resin, it shows significant redshift to $573 \mathrm{~nm}$, wider band width of $55 \mathrm{~nm}$ and secondary peak formation at $610 \mathrm{~nm}$. Signal to noise ratio is also poor due to the low solubility of QDs in PEGDA without compatible surface modification. 